BULL. AUSTRAL. MATH. SOC.

VOL. 31 (1985), 377-387.

\title{
THE SUBVARIETY LATTICE OF THE VARIETY OF DISTRIBUTIVE DOUBLE $p$-ALGEBRAS
}

\author{
WIESLAW DZIOBIAK
}

Let $L$ denote the subvariety lattice of the variety of distributive double $p$-algebras, that is, the lattice whose universe consists of all varieties of distributive double $p$-algebras and whose ordering is the inclusion relation. We prove in this paper that each proper filter in $L$ is uncountable. Moreover, we prove that except for the trivial variety (the zero in $L$ ) and the variety of Boolean algebras (the unique atom in $L$ ) every other element of $L$, generated by a finite algebra, has infinitely many covers in $L$, among which at least one is not generated by any finite algebra. The former result strengthens a result of Urquhart who showed that the lattice $L$ is uncountable. On the other hand, both of our results indicate a high complexity of the lattice $L$ at least in comparison with the subvariety lattice of the variety of distributive $p$-algebras, since a result of lee shows that the latter lattice forms a chain of type $\omega+1$ and every cover in it of the variety generated by a finite algebra is itself generated by a finite algebra.

Received 6 November 1984.

Copyright Clearance Centre, Inc. Serial-fee code: 0004-9727/85 $\$$ A2.00 + 0.00. 


\section{Preliminaries}

An algebra $(A, \wedge, \vee, *,+, 0,1)$ is called a distributive double p-algebra if $(A, \wedge, \vee, 0, \lambda)$ is a bounded distributive lattice and * and ${ }^{+}$are unary operations on $A$ such that $a \wedge x=0$ if and only if $x \leq a^{*}$, and $a \vee x=1$ if and only if $a^{+} \leq x$.

A distributive double $p$-algebra $A$ is said to be of finite range if for each $a \in A$ there exists $n<\omega$ such that $a^{n\left(+^{*}\right)}=a^{(n+1)\left(+^{*}\right)}$, where $a^{O\left(+^{*}\right)}:=a$ and $a^{(k+1)\left(+^{*}\right)}:=a^{k\left(+^{*}\right)+*}$ for every $k \geq 0$. When $a^{n\left(+^{*}\right)}=a^{(n+1)\left(+^{*}\right)}$ for every $a \in A$, the algebra $A$ is said to be of range $n$. A is said to be regular if any two congruences on $A$ with a class in common are equal. By a result of Varlet [11], $A$ is regular if and only if it satisfies the quasi-identity: $x^{*}=y^{*}$ and $x^{+}=y^{+}$imply $x=y$.

With each distributive double p-algebra $A$ there are associated the following sets: $D(A)=\left\{a \in A ; a^{*}=0\right\}, \bar{D}(A)=\left\{a \in A ; a^{+}=1\right\}$ and $\operatorname{Cen}(A)=\left\{a \in A ; a=a^{+*}\right\}$.

A lattice filter $F$ on a distributive double $p$-algebra $A$ is called normal if $a \in F$ implies $a^{+^{*}} \in F$. If $F$ is normal then $C(F)=\{(a, b) \in A \times A ; a \wedge c=b \wedge c$ for some $c \in F\}$ is a congruence relation on $A$.

For a poset $P$, by $\operatorname{Min} P$ and $\operatorname{Max} P$ we denote the set of all minimal and the set of all maximal elements in $P$, respectively. If $X \subseteq P$ then by $[X]$ we denote the set of all $a \in P$ such that $x \leq_{P} a$ for some $x \in X .(X]$ is defined dually. Instead of $[\{x\})$ and $(\{x\}]$ we shall write $[x)$ and $(x]$, respectively. We call a map $f: P \rightarrow Q$ admissible if it is isotone and satisfies $f((x) \cap \operatorname{Min} P)=(f(\dot{x})] \cap \operatorname{Min} Q$ and $f([x) \cap \operatorname{Max} P]=[f(x)) \cap \operatorname{Max} Q$ for every $x \in P$. Recall that the set of all increasing subsets of a poset $P$ with respect to the settheoretical meet and join, and the operations ${ }^{*},{ }^{+}$defined by $X^{*}=P \backslash(X]$ and $X^{+}=[P \backslash X)$ forms a distributive double $p$-algebra. This algebra will be denoted by $D P$.

For a given distributive double p-algebra $A$ let $P(A)$ denote the set of all prime filters on $A$. We have a poset $(P(A), \subseteq)$. The map 
$P: A \rightarrow D P(A)$ defined by $P(a)=\{X \in P(A) ; a \in X\}$ is an embedding of $A$ into $D P(A)$. If $A$ is finite then $P$ is an isomorphism.

Throughout this paper by 2 and 3 we denote, respectively, two and three element chains, considered as distributive double p-algebras. By $V(K), K$ is a class of similar algebras, we denote the least variety containing all algebras of $K$. We shall write $V(A)$ instead of $V(\{A\})$.

For a fuller account concerning distributive double $p$-algebras we refer to [2], [3], [7], [8]. For lattice theory and universal algebra we refer to [4] and [5].

\section{A sequence of finite simple algebras}

In this section we define an infinite sequence of finite simple distributive double p-algebras whose every proper subalgebra is isomorphic to 2 or to 3 . This sequence will be used in proofs of our results.

For each $5 \leq k$ let $P_{k}$ denote a poset with the universe $\{-i ; 1 \leq i \leq k\} \cup\{i ; 1 \leq i \leq k\}$, partially ordered by the relation $\leq_{k}$ defined as follows: for all $x, y, x s_{k} y$ holds if and only if $x=y$, or $x=-y$ and $1 \leq y \leq k$, or $x=-(y-1)$ and $2 \leq y \leq k$, or $-(k-3) \leq x \leq-2$ and $y=k-1$. The diagrams of $P_{5}$ and $P_{6}$ look as follows :
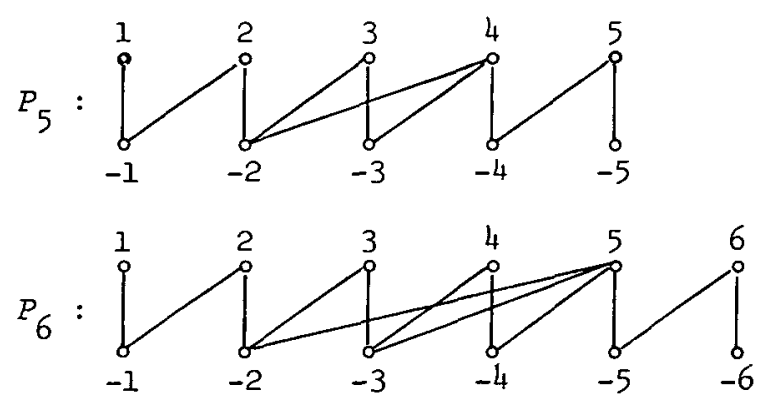

LEMMA 2.1. For each $k$ and a poset $P$, if $f: P_{k} \rightarrow P$ is admissible and not one-to-one then Im $f$ is a 1- or 2-element chain.

Proof. Since $P_{k}$ is connected and $f$ is admissible, it suffices to show that $|\operatorname{Im} f| \leq 2$. 
CLAIM 1. If $1 \leq i \neq j \leq k$ and $f(i)=f(-j)$ then $|\operatorname{Im} f|=1$.

Let $1 \leq i \neq j \leq k$ and $f(i)=f(-j)$. Then $f(i) \in$ Min $P$ since $-j \in \operatorname{Min} P_{k}$. But $f(i) \in \operatorname{Max} P$ because $i \in \operatorname{Max} P_{k}$. Hence $f(i)$ is both minimal and maximal in $P$. Therefore $f(i)=f(x)$ for all $x \in P_{k}$, proving the claim.

For $1 \leq i \leq k$ denote by $N(i)$ the set of all $1 \leq j \leq k$ such that $i \neq j$ and $f(i)=f(j)$ imply $|\operatorname{Im} f| \leq 2$. By induction one can verify the following

CLAIM 2. $N(i)=\{1,2, \ldots, k\} \backslash\{i\}$ for every $1 \leq i \leq k$.

The lemma follows from Claims $l$ and 2 and the observation that if $f(-i)=f(-j)$ for some $1 \leq i \neq j \leq k$ then there exist $1 \leq m \neq n \leq k$ with $f(m)=f(n)$.

LEMMA 2.2 (see [3]). For finite posets $P$ and $Q, D P$ is embeddable into $D Q$ if and only if there exists an admissible map from $Q$ onto $P$.

Proof. The "if" part is a particular case of Proposition 2.2 from Davey [3]. For the "only if" part let $f: D P \rightarrow D Q$ be an embedding. Then the map $g(X)=f^{-1}(X)$ for all $X$, is onto and admissible. Hence the observation that $Q \cong(P(D Q), \subseteq)$ and $P \cong(P(D P), \subseteq)$ concludes the proof.

LEMMA 2.3 (see [3]). For a finite poset $P$,

(i) $D P$ is simple if and only if $P$ is connected and $P=\operatorname{Min} P \cup \operatorname{Max} P$,

(ii) DP is subidrectly irreducible but not simple if and only if $|P|>1, P$ is connected and there exists a unique $x$ of $P$ with $P=(\operatorname{Min} P \cup \operatorname{Max} P) \cup\{x\}$.

COROLLARY 2.4. Each $D P_{k}$ is simple and of range 4 . Moreover, the only, up to isomorphism, proper subalgebras of $D P_{k}$ are 2 and 3 .

Proof. The range of $D P_{k}$ is verified easily. The rest follows from Lemmas $2.1,2.2$ and 2.3 . 


\section{Cardinality of a proper filter in $L$}

We prove here that each proper filter in $L$ is of cardinality $2^{\kappa_{0}}$. We begin with the following lemma.

LEMMA 3.1. Let $K$ be a variety of distributive double p-algebras, all being of the same finite range. Then both the class $k_{s i}$ of subdirectly irreducible algebras in $\mathrm{K}$ and the class $\mathrm{K}_{\mathrm{sim}}$ of simple algebras in $\mathrm{K}$ form universal classes.

Proof. That $K_{s i}$ is a universal class follows directly from a result of Katriñák [8] which says that a distributive double p-algebra $A$ of finite range and with $|D(A)| \geq 2$ is subdirectly irreducible if and only if $|D(A) \cap \bar{D}(A)| \leq 2$ and $\operatorname{Cen}(A)=\{0,1\}$, and from the observation that $A$ is a Boolean algebra if and only if $|D(A)|=1$. That $\mathrm{K}_{\mathrm{sim}}$ forms a universal class is obvious since, by a result of Beazer [1], a nontrivial distributive double $p$-algebra $A$ of range $k$ is simple if and only if $A$ is regular and satisfies $x^{k\left(+^{*}\right)}=0$ for every $x \in A \backslash\{1\}$.

THEOREM 3.2. Each proper filter in $L$ is of cardinality $2^{N_{0}}$.

Proof. It suffices to show that the principal filter in $L$ generated by a proper subvariety $K$ of the variety of distributive double

p-algebras is of cardinality $2^{\kappa_{0}}$. As the variety of distributive double p-algebras is generated by its finite members, there exists a finite poset $P$ having at least two elements and whose distributive double $p$-algebra is subdirectly irreducible and does not belong to $\mathrm{K}$. For each $n \geq 5$, let $P+P_{n}$ denote a poset with universe $P \cup P_{n}$ (we assume here that $P$ and $P_{n}$ are disjoint) and whose ordering is given by $\leq_{P} \cup \leq_{n} \cup\left\{(x, y) ; x \in \operatorname{Min} P_{n}, y \in P, a \leq_{p} y\right.$ and $\left.a \neq y\right\}$ $\cup\left\{(x, y) ; x \in P, y \in \operatorname{Max} P_{n}, x \leq_{P} b\right.$ and $\left.x \neq b\right\}$,

where $a$ and $b$ are fixed elements of $P$ such that $a \in \operatorname{Min} P$, $b \in \operatorname{Max} P$ and $a \leq_{P} b$; of course, such elements exist in $P$ since $|P| \geq 2$ and $D P$ is subdirectly irreducible. We claim that $D P$ is 
embeddable into $D\left(P+P_{n}\right)$ for every $n$. Indeed, in view of Lemma 2.2 , it suffices to consider a map $f: P \cup P_{n} \rightarrow P$ defined by $f(x)=a$ when $x \in \operatorname{Min} P_{n}, f(x)=x$ when $x \in P$ and $f(x)=b$ otherwise.

Now we select inductively a sequence $Q_{0}, Q_{1}, \ldots$ of finite posets as follows: $Q_{0}:=P+P_{5}$ and $Q_{n+1}:=P+P\left|Q_{n}\right|$ for every $n=0,1,2, \ldots$, and, for every $\emptyset \neq I \subseteq \omega$, we define $\mathrm{K}(I):=\mathrm{K} \vee \operatorname{HSP}\left(\left\{D Q_{n} ; n \in I\right\}\right)$. Obviously, $\mathrm{K} \subseteq \mathrm{K}(I)$ for every $I \subseteq \omega$. It remains then to show that $K(I)$ and $K(J)$ are different for different $I$ and $J$. To this end we suppose $K(I)=K(J)$ and consider a fixed $m \in I$. We have $D Q_{m} \in K(J)$ which, in conjunction with Jónsson's Lemma (see [6]), Corollary 2.4 and the above claim, yields that $D Q_{m}$ is a homomorphic image of a subalgebra $A$ of an ultraproduct of some family of algebras from among $D Q_{n}{ }^{\prime} \mathrm{s}$, where $n \in J$. We claim that $D Q_{m}$ is isomorphic to $A$ which would yield that $D Q_{m}$ is embeddable into some $D Q_{n}$, where $n \in J$. Since every $D P_{i}$ is of range 4 and $P$ is finite, then due to the definition of $P+P_{i}$ it follows that all algebras of the form $D Q_{n}$ have the same finite range $k$. If $D P$ is simple then, by Lemma 2.3, every algebra $D Q_{n}$ is simple which, together with Lemma 3.1 , implies that $A$ is simple as well, and thus $D Q_{m}$ is isomorphic to $A$. So assume that $D P$ is not simple. Then, by Lemma 2.3, $D Q_{m}$ is not simple either. Evidently, $A$ is of range $k$ and, by Lemma 3.1, it is subdirectly irreducible which, by Corollary 9 from [2], implies that the lattice of congruence relations on $A$ is a 2- or 3-element chain. From this, since $D Q_{m}$ is not simple, it follows that $D Q_{m}$ is isomorphic to $A$, completing the proof of the claim. As $D Q_{m}$ is finite, then by the claim we conclude that $D Q_{m}$ is embeddable into some $D Q_{n}$, where $n \in J$. Hence, by Lemma 2.2, there exists an admissible map $f$ from $Q_{n}$ onto $Q_{m}$. We show $m=n$. To the contrary suppose $m \neq n$. Then $m<n$, 
since $f$ is onto, and hence $\left|Q_{m}\right|<\left|P_{Q_{n-1}}\right| \mid$. Therefore, the map $f \uparrow P\left|Q_{n-1}\right|$ is not one-to-one which, together with Lemma 2.1 , yields $\left|\operatorname{Im} f \uparrow P_{\mid Q_{n-1}}\right| \mid \leq 2$. So, we have

$$
\left|Q_{m}\right|=|\operatorname{Im} f \uparrow P \cup \operatorname{Im} f \uparrow P| Q_{n-1}|| \leq|P|+2,
$$

a contradiction. Thus $m=n$, and so $m \in J$ which proves $I \subseteq J$. Similarly, we show $J \subseteq I$. Thus $I=J$, concluding the proof.

\section{On the bottom part of $L$}

In this section we will be concerned with the bottom part of $L$. For our efforts the following two lemmas are crucial.

LEMMA 4.1. Let $A$ be a subdirectly irreducible distributive double p-algebra with $|A| \geq 3$. Then there exist a set $I$ and a congruence relation $\theta \neq Z$ on $A^{I}$ such that $D\left(A^{I} / \theta\right) \cap \bar{D}\left(A^{I} / \theta\right) \neq \emptyset$.

Proof. Since $A$ is subdirectly irreducible, then by Theorem 4 from Katriňák [8], $\operatorname{Cen}(A)=\{0,1\}$. This yields $x \vee x^{*} \in D(A) \backslash\{1\}$ for every $x \in A \backslash\{0,1\}$. Hence $D(A) \backslash\{1\} \neq \varnothing$. On the other hand, by Lemma 7 from Katriñák [8], $\wedge\left(x^{n\left(+^{*}\right)} ; n<\omega\right)=0$ for all $x \in D(A) \backslash\{1\}$. Therefore, in the case when $A$ is of finite range, we have $x^{n\left(+^{*}\right)}=0$ for some $x \in D(A)$ and an integer $n$. But this, in view of the corollary succeeding Theorem 7 from Katrinăk [8], implies that $D(A) \cap \bar{D}(A)$ is nonempty. So, in the case when $A$ is of finite range, it suffices to take $I=\{0\}$ and $\theta=\mathrm{id}$.

Let $A$ be not of finite range. Then, for a certain element $a$ of $A$, the sequence $a^{n\left(+^{*}\right)}, n<\omega$, is strictly descending. Put $b_{n}:=a \wedge a^{2(n+1)\left(+^{*}\right)+}$ and $c_{n}:=\vee\left(a^{j\left(+^{*}\right)} \wedge a^{j\left(+^{*}\right)+} ; j \leq 2(n+1)\right)$ where $n<\omega$.

CLAIM 1. $b_{n} \wedge c_{n}^{*}=0$ for every $n<\omega$.

Proof. It suffices to prove that $P\left(b_{n}\right) \cap P\left(c_{n}^{*}\right)=\emptyset$. But each prime filter on $A$ is contained in some maximal prime filter, so in fact it is 
enough to prove that for each maximal prime filter $X$ on $A$ the following condition holds: $X \in P\left(b_{n}\right)$ if and only if $X \in P\left(c_{n}\right)$. The "if" part directly follows from the observation that $c_{n} \leq b_{n}$. For the "only if" part, let $X \in P\left(b_{n}\right)$ be maximal. Then $X \in P(a)$ and $X \notin P\left(a^{(2(n+1)+1)\left(+^{*}\right)}\right)$ because $a^{2(n+1)\left(+^{*}\right)+\wedge a^{(2(n+1)+1)(+*)}}=0$. Hence, there exists a greatest $k<\omega$ such that $X \in P\left(a^{k(+*)}\right)$ and $X \notin P\left(a^{(k+1)\left(+^{*}\right)}\right)$. Since $X \notin P\left(a^{(k+1)\left(+^{*}\right)}\right), \quad X \notin P(A) \backslash\left(P\left(a^{k\left(+^{*}\right)+}\right)\right]$. Hence $X \in\left[P\left(a^{k\left(+^{*}\right)+}\right)\right]$ and, therefore, $X \in P\left(a^{k(+*)+}\right)$ because $X$ is maximal. Thus $X \in P\left(a^{k\left(+^{*}\right)} \wedge a^{k\left(+^{*}\right)+}\right)$ which, by $k \leq 2(n+1)$, implies $x \in P\left(c_{n}\right)$.

CLAIM 2. $b_{n} \wedge c_{n}^{+}=b_{n}$ for every $n<\omega$.

Proof. $\bar{D}(A)$ is an ideal on $A$ and $a^{j\left(+^{*}\right)} \wedge a^{j\left(+^{*}\right)+} \epsilon \bar{D}(A)$. Hence $c_{n} \in \bar{D}(A)$, and so $b_{n} \wedge c_{n}^{+}=b_{n}$.

CLAIM 3. $b_{n}^{n\left(+^{*}\right)} \neq 0$ for every $n<\omega$.

Proof. For $j, m$ we have

$$
\begin{aligned}
& a^{(j+1)\left(+^{*}\right)} \wedge a^{(m-1)\left(+^{*}\right)+} \leq a^{(j+1)\left(+^{*}\right)} \wedge a^{(m-1)\left(+^{*}\right)+* *} \\
& \quad=a^{(j+1)\left(+^{*}\right)} \wedge a^{m\left(+^{*}\right) *} \leq a^{(j+1)\left(+^{*}\right)} \wedge a^{m\left(+^{*}\right)++^{*}}=\left(a^{j\left(+^{*}\right)} \wedge a^{m\left(+^{*}\right)++^{*}} .\right.
\end{aligned}
$$

Moreover, $a^{j\left(+^{*}\right)} \wedge a^{j\left(+^{*}\right)+} \neq 0$ for every $j<\omega$ because the sequence $a^{j\left(+^{*}\right)}, j<\omega$, is strictly descending. Hence $b_{n}^{n\left(+^{*}\right)} \neq 0$ for every $n<\omega$.

Let $b=\left(b_{n} ; n<\omega\right)$ and $c=\left(c_{n} ; n<\omega\right)$. By Claim 3 , the normal filter $F$ on $A^{\omega}$ generated by $b$ is proper. So $C(F) \neq l$. But, by Claims 1 and 2, $[C] C(F) \in D\left(A^{\omega} / C(F)\right) \cap \bar{D}\left(A^{\omega} / C(F)\right)$, proving the lemma.

LEMMA 4.2. Let $U$ be a non-principal ultrafizter on $w \backslash\{0,1,2,3,4\}$. Then 2 and 3 are, up to isomorphism, the only finite subdirectly irreducible algebras in $V\left(\Pi_{U}\left(D P_{k} ; 5 \leq k<\omega\right)\right)$. 
Proof. That 2 and 3 are subdirectly irreducible and belong to $V\left(\Pi_{U}\left(D P_{k} ; 5 \leq k<\omega\right)\right)$ is obvious. Let $A \in V\left(\Pi_{U}\left(D P_{k} ; 5 \leq k<\omega\right)\right)$ be finite and subdirectly irreducible. Then, by Jónsson's Lemma, $A \in \operatorname{HSP}_{U}\left(\left\{\Pi_{U}\left(D P_{k} ; 5 \leq k<\omega\right)\right\}\right)$. By Corollary 2.4 and Lemma 3.1 , each algebra belonging to $\operatorname{SP}_{U}\left(\left\{\Pi_{U}\left(D P_{k} ; 5 \leq k<\omega\right)\right\}\right)$ is simple. From this we get that $A$ is simple and, by the assumption that $A$ is finite, we conclude that $A$ is embedded into some direct power of $\Pi_{U}\left(D P_{k} ; 5 \leq k<\omega\right)$. This yields that $A$ is embeddable into $I_{U}\left(D P_{k} ; 5 \leq k<\omega\right)$. Therefore there exists $I \in U$ such that $A$ is isomorphic to a subalgebra of $\Pi\left(D P_{k} ; k \in I\right)$; here we used again the assumption that $A$ is finite. Since $U$ is non-principal and $A$ is simple, then by Corollary 2.4 we get that $A$ is isomorphic to 3 or to 2 .

THEOREM 4.3. (i) $V(3)$ is a unique cover of $V(2)$ in $L$.

(ii) $V(3)$ has infinitely many covers in $L$, among which at least one is not generated by any finite algebra.

Proof. (i) That $V(3)$ covers $V(2)$ is obvious. That $V(3)$ is unique follows from Lemma 4.1 .

(ii) By Corollary 2.4, every variety $V\left(D P_{k}\right)$, where $5 \leq k<\omega$, covers $V(3)$. To prove the rest, let $U$ be a fixed non-principal ultrafilter on $\omega \backslash\{0,1,2,3,4\}$. We have $V(3) \varsubsetneqq V\left(\pi_{U}\left(D P_{k} ; 5 \leq k<\omega\right)\right)$.

So, as $V(3)$ is finitely based, there exists a cover $K$ of $V(3)$ contained in $V\left(\Pi_{U}\left(D P_{k} ; 5 \leq k<\omega\right)\right)$. By Lemma $4.2, k$ is not generated by any finite algebra.

THEOREM 4.4. For a variety $K$ generated by a finite distributive double p-algebra the following conditions are equivalent:

(i) every variety that covers $K$ in $L$ is generated by a finite algebra;

(ii) $\mathrm{K}$ has finitely many covers in $L$;

(iii) $\mathrm{K}$ is the trivial variety or the variety of Boolean algebras. 
Proof. By Theorem 4.3, (iii) implies (i) and (ii). It remains then to show that both (i) and (ii) imply (iii).

(i) $\Rightarrow$ (iii). Suppose that $K$ is a nontrivial variety and $K \neq V(2)$. Then, by Theorem $4.3, V(3) \subseteq K$. Let $L$ be a fixed variety not generated by any finite algebra that covers $V(3)$ in $L$; by Theorem 4.3 , such a cover really exists. We claim that $V(K \cup L)$ covers $K$ in $L$, and that $V(K \cup L)$ is not generated by any finite algebra. The latter claim is immediate since otherwise, by Jónsson's Lemma, $L$ would be generated by a finite algebra. To prove the former one, let $K \subseteq M \subseteq V(K \cup L)$. Then, by Jónsson's Lemma, $K_{s i} \subseteq M_{s i} \subseteq K_{s i} \cup L_{s i}$. When $M_{s i} \cap L_{s i} \subseteq I(\{2,3\})$, then $M_{s i}=K_{s i}$, and so $M=K$. Otherwise, $A \in M_{s i} \cap L_{s i}$ for some $A \nsubseteq 2$ and $A \neq 3$. But $L$ covers $V(3)$, so $L=V(\{3, A\})$. Hence $\mathbf{M}=V(K \cup L)$. Obviously, $\mathrm{K} \neq V(\mathrm{~K} \cup \mathrm{L})$. Thus $V(\mathrm{~K} \cup \mathrm{L})$ covers $\mathrm{K}$ in $L$.

(ii) $\Rightarrow$ (iii). Suppose that $K$ is nontrivial and $K \neq V(2)$. Then $V(3) \subseteq \mathrm{K}$ by Theorem 4.3. Let $n$ be a least natural number such that $|A|<n$ for every subdirectly irreducible algebra $A$ from $K$. Clearly, such a number exists because $K$ is generated by a finite algebra and, by Jónsson's Lemma, every subdirectly irreducible algebra in $K$ is finite and there are, up to isomorphism, only finitely many of them. Notice that $K \neq V\left(K \cup\left\{D P_{k}\right\}\right)$ for every $n \leq k$. Moreover, by Corollary 2.4, $V\left(K \cup\left\{D P_{k}\right\}\right)$ covers $K$ in $L$, and $V\left(K \cup\left\{D P_{k}\right\}\right) \neq V\left(K \cup\left\{D P_{m}\right\}\right)$ for all $n \leq k \neq m$, proving that (ii) implies (iii).

\section{References}

[1] R. Beazer, "The determination congruence on double p-algebras", Algebra Universalis 6 (1976), 121-129.

[2] R. Beazer, "Subdirectly irreducibles for various pseudocomplemented algebras", Algebra Universalis 10 (1980), 225-231.

[3] Brian A. Davey, "Subdirectly irreducible distributive double p-algebras", Algebra Universalis 8 (1978), 73-88. 
[4] George Grätzer, General lattice theory (Mathematische Reihe, 52. Birkhauser Verlag, Basel, Stuttgard, 1978).

[5] George Grätzer, Universal algebra (Springer Verlag, New York, Heidelberg, Berlin, 1979).

[6] Bjarni Jónsson, "Algebras whose congruence lattices are distributive", Math. Scand. 21 (1967), 110-121.

[7] T. Katriñák, "The structure of distributive double p-algebras. Regularity and congruences", Algebra Universalis 3 (1973), $238-246$.

[8] T. Katriñák, "Subdirectly irreducible distributive double p-algebras", Algebra Universalis 10 (1980), 195-219.

[9] K.B. Lee, "Equational classes of distributive pseudo-complemented lattices", Canad. J. Math. 22 (1970), 881-891.

[10] Alasdair Urquhart, "Equational classes of distributive double p-algebras", Algebra Universalis 14 (1982), 235-243.

[11] J.C. Varlet, "A regular variety of type $(2,2,1,1,0,0)$ ", Algebra Universalis 2 (1972), 218-223.

Section of Logic,

Polish Academy of Sciences,

Kódź,

Piotrkowska 179 ,

Poland.

Correspondence address:

Institute of Mathematics,

N. Copernicus University,

ioruń,

Poland. 